\title{
Estapedostomía endoscópica: Experiencia en el Hospital del Salvador
}

\section{Endoscopic Stapedotomy: Experience at the Hospital del Salvador}

\author{
Roberto Arias A $^{1}$, Mauricio Silva $C^{1}$, Marcela Veloz T².
}

\section{RESUMEN}

Introducción: La otoesclerosis es una enfermedad metabólica, localizada y primaria del hueso de la cápsula ótica y la cadena osicular. Produce fijación de la cadena y consecuente hipoacusia de conducción.

Objetivo: El objetivo del presente trabajo es mostrar la estapedostomía endoscópica como alternativa quirúrgica, y comparar sus resultados con la estapedostomía microscópica.

Material y método: Estudio restrospectivo de revisión de fichas clínicas de pacientes intervenidos quirúrgicamente en nuestro servicio desde enero de 2012 hasta de junio de 2014 con sospecha de otoesclerosis. Se obtuvieron un total de 35 pacientes operados, 25 vía microscópica y 10 vía endoscópica.

Resultados: El tiempo operatorio promedio fue de 60 y 85 minutos para microscópica y endoscópica, respectivamente. El cierre de diferencia óseo aérea (gap), a los 2 meses, fue de 100\% para endoscópica y de 92\% para microscópica. Un paciente vía endoscópica y 2 pacientes vía microscópica presentaron disgeusia.

Conclusiones: La estapedostomía endoscópica es una alternativa quirúrgica ya que permite una excelente visión y comprensión de la anatomía. Su mayor utilidad es que permite trabajar con mejor exposición en conductos auditivos externos (CAE) estrechos y angulados. También contribuye a disminuir el fresado del CAE con menor daño de la cuerda del tímpano. Sus resultados auditivos son comparables con la técnica con microscopio.

Palabras clave: Estapedostomía, endoscópica, microscópica, anatomía.

\begin{abstract}
Introduction: Otosclerosis is a metabolic bone disease of the otic capsule and the ossicles. It is a primary disease that may cause fixation of the stape and conductive hearing loss.
\end{abstract}

Médico Servicio de Otorrinolaringología, Hospital del Salvador. 
Aim: To show endoscopic stapedotomy as a surgical alternative, and to compare the results with the stapedotomy using microscope.

Material and method: Clinical records were reviewed of patients treated surgically in our department from January 2012 to June 2014 with suspected otosclerosis. 35 patients were obtained, 25 using microscope and 10 using only endoscope.

Results: Average operative time was 60 and 85 minutes to microscopic and endoscopic group respectively. Air-bone gap closureat 2 months was 100\% for endoscopic and $92 \%$ for microscopic surgery. One patient of endoscopic surgery and two patients of microscopic surgery presented postsurgical dysgeusia.

Conclusions: Endoscopic stapedotomyis a surgical alternative as it allows an excellent vision and understanding of anatomy; it can be performeddespite a curved and narrowexternalauditory canal (EAC), with a betterexposure.lt also helps to reduce the milling of EAC with less damage to the chorda tympani. Hearing result is comparable to the microscope surgery.

Key words: Stapedotomy, endoscopic, microscopic, anatomy.

\section{INTRODUCCIÓN}

La otoesclerosis es una enfermedad metabólica, localizada y primaria del hueso de la cápsula ótica y la cadena osicular, caracterizada por reabsorción asociada a neoformación ósea. El proceso de otoespongiosis se inicia primariamente en el estribo, causando hipoacusia de conducción. Se puede presentar como hipoacusia mixta si este proceso se extiende hacia la cóclea ${ }^{1}$. Es una enfermedad autosómica dominante con baja penetrancia y expresión variable, que se presenta habitualmente entre los 40 y 50 años de edad y con mayor frecuencia en las mujeres ${ }^{1,2}$.

La otoesclerosis se presenta la mayoría de las veces con una hipoacusia lentamente progresiva, bilateral en el $80 \%$ de los casos y asimétrica. A la otoscopía el tímpano suele encontrarse normal 0 presentar una coloración roja sobre el promontorio o la zona anterior a la ventana oval, conocida como el signo de Schwartze. En la audiometría se observa clásicamente una hipoacusia de conducción, o mixta si hay compromiso coclear. Puede presentarse el signo «Notch de Carhart» que consiste en una baja en la vía ósea en las frecuencias de los 2.000 $\mathrm{Hz}$ acercándose a la vía aérea. La impedanciometría puede mostrar curva $A$ o $A_{s}$ y reflejos negativos, efecto on-off y reflejos invertidos ${ }^{3}$. La tomografía computarizada de oídos es un apoyo para el diagnóstico diferencial y la planificación de la cirugía.

El manejo quirúrgico de la otoesclerosis a nivel del estribo se inicia en la década de 1950 con la realización de las primeras estapedectomías. Las técnicas quirúrgicas han ido evolucionando permitiendo menor trauma y mejor resultado en estos pacientes ${ }^{4}$, siendo la más utilizada actualmente la estapedostomía (fenestración de la patina del estribo) e instalación de prótesis.

En Chile, se han reportado excelentes resultados auditivos y baja tasa de complicaciones en pacientes con otoesclerosis bilateral (operados uni o bilateralmente) y unilateral sometidos a estapedostomía desde la década de $1980^{5,6}$. También se ha estudiado el grado de afectación histológico de la platina del estribo y los umbrales audiométricos prequirúrgicos encontrándose que no existe correlación entre éstos, y tampoco con el aspecto macroscópico del estribo. Tampoco se encontró relación entre el grado histológico y la mejoría de vía aérea posquirúrgica y el cierre de gap osteo-aéreo en estos pacientes? ${ }^{7}$.

La estapedostomía es considerada una cirugía de alta precisión realizada habitualmente bajo visión microscópica. Desde la década de los noventa se ha introducido la cirugía endoscópica de oído (CEO) para timpanoplastías, osiculoplastías, cirugía de colesteatoma, implante coclear e incluso neurinomas del acústico ${ }^{8-13}$. En el año 2000 se describe el uso de CEO para estapedectomías ${ }^{14}$.

\section{OBJETIVO}

Este trabajo tiene como objetivo mostrar la estapedostomía por vía endoscópica como una 
alternativa quirúrgica y comparar nuestros resultados con la estapedostomía vía microscopio.

\section{MATERIAL Y MÉTODO}

Se realizó un estudio restrospectivo de revisión de fichas clínicas de pacientes intervenidos quirúrgicamente, mediante vía microscópica y endoscópica, por el mismo cirujano, en el Servicio de Otorrinolaringología (ORL) del Hospital del Salvador desde enero de 2012 hasta junio de 2014 con sospecha clínica de otoesclerosis. Se obtuvo un total de 35 pacientes operados, 25 vía microscópica y 10 mediante vía endoscópica. Se analizaron los datos usando cifras absolutas, porcentuales y promedios.

Se consideraron los siguientes criterios de inclusión:

- Historia clínica compatible

- Otoscopía normal

- Audiometría con hipoacusia de conducción con gap $\geq 30 \mathrm{~dB}$

- Impedanciometria con curvas A o As

- Tomografía computarizada de oídos con protocolo de otoesclerosis (cortes de 0,6 mm)

- Sin historia de otitis media crónica

Se excluyó a pacientes con gap menor 30.

El equipamiento quirúrgico empleado en las cirugías estaba compuesto por un endoscopio rígido $S t o r z^{\circledR}$ de $4 \mathrm{~mm}$ y $2,7 \mathrm{~mm}$ de $0^{\circ}$, de $14 \mathrm{~cm}$; una torre de video $S$ torz ${ }^{\circledR}$ con cámara full HD; un Skeeter drill ${ }^{\circledR}$ de Medtronic con fresa de 0,8 mm y una consola Integrated Power Console de Medtro$n i c^{\circledR}$ (IPC system).

En todos los pacientes se utilizó una prótesis tipo Schuknecht de fluoroplastic de $0,6 \mathrm{~mm}$ de diámetro y de 4,5 mm de largo.

\section{Técnica quirúrgica}

Bajo anestesia total intravenosa (TIVA, por sus siglas en inglés) se posiciona al paciente en decúbito dorsal con hombro ipsilateral al oído a intervenir más debajo de la posición de su cabeza. La cirugía se lleva a cabo con el cirujano sentado con ambos codos apoyados en mesa quirúrgica con torre endoscópica al frente.
Se colocan cotonoides en el CAE con vasoconstrictor y se infiltra con lidocaína/epinefrina al 1:100.000 en CAE por vía endoaural.

Bajo visión endoscopica de $0^{\circ}$ se realiza incisión vía endomeatal en forma de rectángulo de las 12 a las 6 horas, paralela al annulus y a una distancia mayor de $4 \mathrm{~mm}$ de éste, donde termina la porción cartilaginosa del CAE y comienza la ósea a modo de obtener un adecuado colgajo tímpano meatal.

Se levanta un colgajo tímpano meatal cuidando no perforar el tímpano, se moviliza y preserva la cuerda del tímpano.

Bajo visión endoscópica se pueden observar las siguientes estructuras: articulación incudoestapedial, las cruras del estribo, el tendón del músculo del estribo, la eminencia piramidal, el nicho de la ventana redonda, el promontorio, el sinus timpani, el canal semicircular lateral, la porción timpánica del nervio facial, el subiculum y el ponticulum (Figura 1).

Se realiza fresado o curetaje de la pared póstero superior del CAE de ser necesario, para lograr mejor exposición.

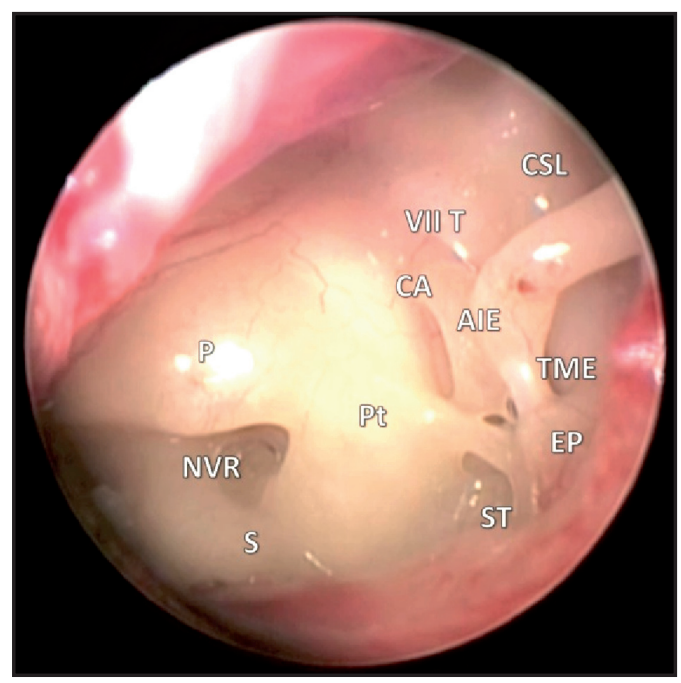

Figura 1. Visión endoscópica del mesotímpano de uno de los pacientes intervenidos por vía endoscópica. P, promontorio; NVR, nicho ventana redonda; S, subiculum; Pt, ponticulum; ST, sinus timpani; AIE, articulación incudoestapedial; CA, crura anterior; VII T, porción timpánica del nervio facial; CSL, canal semicircular lateral; TME, tendón del músculo del estribo; EP, eminencia piramidal. 


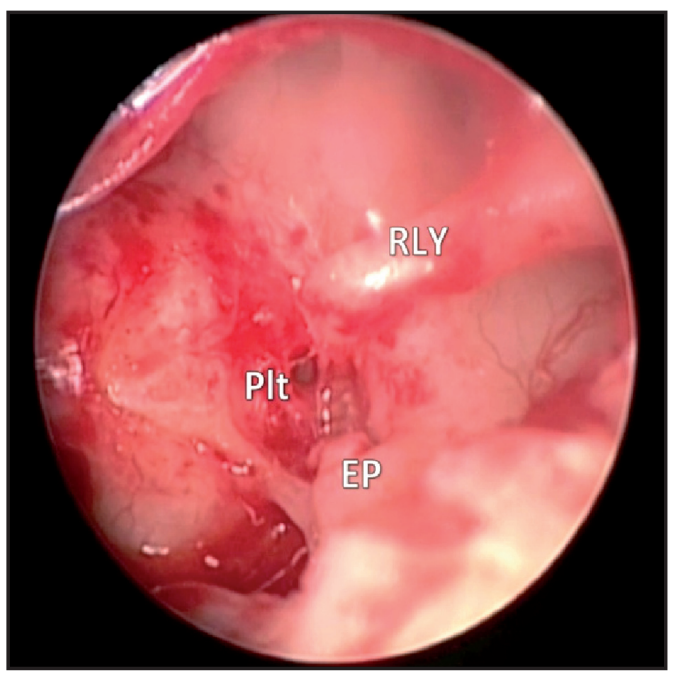

Figura 2. Platinostomía de uno de los pacientes intervenidos por vía endoscópica. La supraestructura del estribo ya ha sido resecada. PIt, platinostomía; EP, eminencia piramidal; RLY, rama larga del yunque.

Los márgenes de seguridad son: por superior el nervio facial, por posterior la eminencia piramidal y por inferior la ventana redonda.

Se realiza la platinostomía con fresa de $0,8 \mathrm{~mm}$ con el Skeeter drill ${ }^{\circledR}$, preservando la supraestructura. Luego se secciona del músculo del estribo ojalá lo más cerca de la pirámide, se fracturan ambas cruras del estribo hacia el promontorio y se remueve la supraestructura de estribo (Figura 2).

Luego se realiza la medición de la prótesis. Se instala prótesis tipo Schuknecht generalmente de 4,5 mm (o 4,25 o 4,75 mm) cuyo gancho se ajusta a la rama larga del yunque (Figura 3 ). Hay que recordar que el sáculo está a $1 \mathrm{~mm}$ y el utrículo a 1,5 $\mathrm{mm}$ de la platina por lo que la prótesis debe quedar inserta en forma precisa.

Finalmente se reposiciona colgajo tímpano meatal que se deja cubierto con gelfoam. La técnica es similar a realización por vía microscopio.

\section{RESULTADOS}

Se revisaron un total de 10 fichas clínicas de estapedostomía endoscópica, 8 correspondieron a mujeres y 2 a hombres. La edad promedio fue de 45,3 años (rango de edad 32 a 59 años). Se

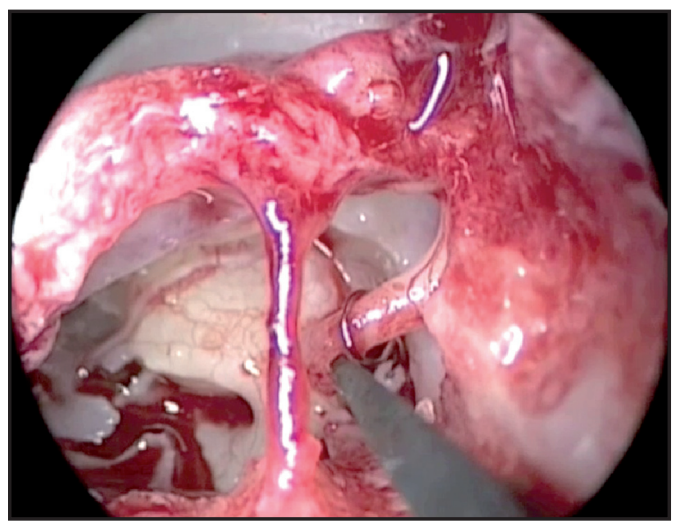

Figura 3. Colocación de la prótesis tipo Schuknecht de 4,5 mm de uno de los pacientes intervenidos por vía endoscópica.

revisaron las audiometrías pre y posoperatorias, esta última se realizó 62 días después de la cirugía en promedio (rango 54 a 70 días). Todos los pacientes presentaron mejoría del PTP aéreo, con un promedio de mejoría de 29,4 dB (rango 20 a $34,7 \mathrm{~dB}$ ), y cierre de gap osteo-aéreo $<10 \mathrm{~dB}$ en 9 pacientes y entre 10 y $20 \mathrm{~dB}$ en 1 paciente. Los resultados se muestran en la Tabla 1.

En el posoperatorio inmediato 3 pacientes del grupo estapedostomía endoscópica $(30 \%)$ presentaron mareos. En este mismo grupo, solo un paciente presentó disgeusia y no se encontraron pacientes con parálisis facial posquirúrgica.

Un total de 25 pacientes fueron intervenidos vía microscópica, con promedio de edad de 48,2 años. Se revisaron sus audiometría pre y posoperatorias. Su control posoperatorio fue realizado después de los 2 meses. Los resultados se muestran en la Tabla 2.

Al comparar los resultados de la cirugía endoscópica versus la cirugía microscópica, el porcentaje de pacientes que lograron cierre de gap osteo-aéreo fue menor en vía microscópica. Se observa un porcentaje similar de pacientes con disgeusia después de 2 meses de seguimiento. Lo mismo ocurre con la presencia de mareos en el posoperatorio inmediato; aunque estas comparaciones se encuentran limitadas por la diferencia en el número de pacientes intervenidos por vía endoscópica versus microscópica. Los resultados se muestran en la Tabla 2. 
Tabla 1. Resultados cirugía endoscópica: tiempo operatorio, cierre de gap y variación de PTP poscirugía

\begin{tabular}{|cccccc|}
\hline Paciente & Tiempo operatorio & Cierre de gap & PTP aéreo pre & PTP aéreo post & Variación PTP \\
\hline 1 & 105 & $<10$ & 68 & 33,3 & 34,7 \\
2 & 135 & $<10$ & 60 & 31,5 & 28,5 \\
3 & 910 & $<10$ & 68 & 23 & 45 \\
4 & 10 & $<10$ & 60 & 35 & 25 \\
5 & 610 & 48 & 22 & 26 \\
6 & 80 & $<10$ & 60 & 25 & 25 \\
7 & 85 & $<10$ & 70 & 30 & 30 \\
8 & 70 & 10 a 20 & 55 & 35 & 35 \\
9 & 75 & - & 65 & 45 & 25 \\
10 & 65 & 60,4 & 30,98 & 29,42 \\
\hline
\end{tabular}

Tabla 2. Comparación entre cirugía endoscópica y microscópica: tiempo quirúrgico, cierre de gap, disgeusia y mareos

\begin{tabular}{|lll|}
\hline & Cirugía endoscópica & Cirugía microscópica \\
\hline Tiempo quirúrgico $(\mathrm{min})$ & 85 & 60 \\
Cierre de gap & $100 \%(10)$ & $92 \%(55)$ \\
Disgeusia & $10 \%(1)$ & $8 \%(2)$ \\
Mareos & $30 \%(3)$ & $28 \%(7)$ \\
N & 10 & 25 \\
\hline
\end{tabular}

\section{DISCUSIÓN}

Desde sus inicios, la estapedostomía se ha realizado mediante el uso de microscopio utilizando ambas manos. La CEO ha sido utilizada desde la década del noventa en niños y adultos, principalmente para cirugía de colesteatoma y timpanoplastías, inicialmente para asistir cirugías con microscopio y mejorar la visualización de áreas anatómicas difíciles de observar con este último (cirugía microscópica de oído asistida por endoscopía) y posteriormente como una técnica exclusivamente endoscópica ${ }^{8-11,13,15}$.

En los ultimos años se ha introducido el uso de CEO para estapedostomías, con resultados similares a la cirugía convencional con microscopio en el resultado auditivo y complicaciones ${ }^{16,17}$.

La CEO permite un ángulo de visión más amplio, mejor visualización de estructuras que son paralelas al microscopio, visión completa del tímpano y mejor comprensión de la anatomía. Además utiliza una vía de acceso natural, como el conducto auditivo externo (CAE). Permite visualizar regiones anatómicas escondidas, como el epitímpano, retrotímpano, y recesos profundos, y es una cirugía mínimamente invasiva con menor riesgo de sangrado, intra y posoperatorio ${ }^{18-20}$.

La estapedostomía por vía endoscópica permite una excelente visión de la anatomía del oído medio y es de gran utilidad sobre todo en pacientes con conductos auditivos estrechos y angulados. Esto podría disminuir la necesidad de fresado de la pared posterosuperior del CAE y con ello el daño sobre la cuerda del tímpano ${ }^{16,17}$.

Dentro de las desventajas se describe la pérdida de la percepción de profundidad y visión binocular, es una cirugía de "una mano" y requiere de un campo sin sangre, de endoscopios de varios diámetros y de un entrenamiento adecuado. La pérdida de visión estereoscópica ha sido considerada importante al momento de fracturar las cruras estapediales, lo cual podría parecer menos relevante al aumentar el número de procedimientos realizados ${ }^{18}$. 
Considerando el bajo número de pacientes intervenidos vía endoscópica en comparación con la cirugía microscópica, nuestros resultados nos orientan a que no existe una diferencia en el resultado auditivo (cierre de gap) ni en el tiempo quirúrgico. En este estudio la estapedostomía endoscópica parece ser una cirugía segura, con una incidencia de complicaciones similar a la cirugía microscópica.

\section{CONCLUSIONES}

Este estudio posee las limitaciones propias del pequeño número de pacientes operados, del tiempo de seguimiento de los pacientes y de la diferencia en el número de pacientes intervenidos por vía endoscópica versus cirugía microscópica.

Se requiere una mayor cantidad de pacientes sometidos a espedostomía endoscópica y un seguimiento a más largo plazo de estos pacientes para poder determinar si esta cirugía resulta en un similar o mejor resultado auditivo y menos complicaciones en comparación con la estapedostomía microscópica.

Como toda nueva técnica quirúrgica tiene su curva de aprendizaje y un tiempo de adquisición de habilidades. Sin embargo consideramos que es una excelente alternativa quirúrgica con gran utilidad docente debido a una mejor visualización, y por ende, a una mejor comprensión de la compleja anatomía del oído. Su elección definitiva depende de la experiencia del equipo quirúrgico.

\section{BIBLIOGRAFÍA}

1. FLINT PW. Cummings otolaryngology--head \& neck surgery. Sixth edition. ed.

2. Carlos Stott C PaJ, Leonor Moyano S. ¿Qué sabemos de otoesclerosis? Aspectos anatomopatológicos. Rev Otorrinolaringol Cir Cabeza Cuello 2005; 65: 179-86.

3. Goycoolea M PM, Shumrick D, Gluckman J. ed Otoesclerosis. En: Otology and Neurotology 1991.

4. RIZER FM, LIPPY WH. Evolution of techniques of stapedectomy from the total stapedectomy to the small fenestra stapedectomy.
Otolaryngologic Clinics of North America 1993; 26(3): 443-51.

5. Délano P AA, Ojeda A, Stott C. Resultados auditivos y hallazgos quirúrgicos en pacientes con cirugía bilateral por otoesclerosis. Rev Otorrinolaringol Cir Cabeza Cuello 2011; 71: 201-6.

6. Stott C nR, Manieu D. Manejo quirúrgico en otoesclerosis unilateral. Rev Otorrinolaringol Cir Cabeza Cuello 2005; 65: 111-6.

7. Stott C OA, Muñoz D, Moyano L. Otoesclerosis. Aspectos histopatológicos y resultados auditivos de la estapedostomía. Rev Otorrinolaringol Cir Cabeza Cuello 2012; 72: 125-32.

8. Dundar R, Kulduk E, Soy FK, et al. Endoscopic versus microscopic approach to type 1 tympanoplasty in children. International Journal of Pediatric Otorhinolaryngology 2014; 78(7): 1084-9.

9. Lima Td0, Araújo TFd, Soares lCA, Testa JRG. Impacto da endoscopia no tratameno do colesteatoma. Brazilian Journal of Otorhinolaryngology 2013; 79: 505-11.

10. TARABICHI M. Endoscopic transcanal middle ear surgery. Indian Journal of Otolaryngology and Head and Neck Surgery: Official publication of the Association of Otolaryngologists of India 2010; 62(1): 6-24.

11. Kakehata $S$, Futal K, Sasaki A, Shinkawa $H$. Endoscopic transtympanic tympanoplasty in the treatment of conductive hearing loss: Early results. Otology \& Neurotology 2006; 27(1): 14-19.

12. Migirov L, Shapira Y, Wolf M. The feasibility of endoscopic transcanal approach for insertion of various cochlear electrodes: a pilot study. Eur Arch Otorhinolaryngol 2014.

13. Aokı K. Advantages of endoscopically assisted surgery for attic cholesteatoma. Diagnostic and Therapeutic Endoscopy 2001; 7(3-4): 99-107.

14. PoE DS. Laser-assisted endoscopic stapedectomy: a prospective study. The Laryngoscope 2000; 110(5 Pt 2 Suppl 95): 1-37.

15. TARABICHI M. Transcanal endoscopic management of cholesteatoma. Otology \& Neurotology: Official publication of the American Otological Society, American Neurotology Society [and] European Academy of Otology and Neurotology 2010; 31(4): 580-8. 
16. Kojima H, Komori M, Chikazawa S, et al. Comparison between endoscopic and microscopic stapes surgery. The Laryngoscope 2014; 124(1): 26671.

17. Nogueira Júnior JF, Martins MJB, Aguiar CV, Pinhelro Al. Estapedotomia totalmente endoscópica: técnica e resultados preliminares. Brazilian Journal of Otorhinolaryngology 2011; 77: 721-7.

18. Badr-El-Dine M, James AL, Panetti G, Marchion D, Presutti L, Nogueira JF. Instrumentation and technologies in endoscopic ear surgery. Otolaryngologic Clinics of North America 2013; 46(2): 211-25.

19. Tarabichi M, Marchioni D, Presutti L, Nogueira JF, PotHier D. Endoscopic transcanal ear anatomy and dissection. Otolaryngologic Clinics of North America 2013; 46(2): 131-54.

20. РотнIER DD. Introducing endoscopic ear surgery into practice. Otolaryngologic Clinics of North America 2013; 46(2): 245-55. 